\title{
Experimental instruction in photonics for high school students: approaches to managing problems faced
}

\section{Zhengyang Choong}

Zhengyang Choong, "Experimental instruction in photonics for high school students: approaches to managing problems faced," Proc. SPIE 10452, 14th Conference on Education and Training in Optics and Photonics: ETOP 2017, 104524B (16 August 2017); doi: 10.1117/12.2266605

SPIE Event: 14th Conference on Education and Training in Optics and Photonics, ETOP 2017, 2017, Hangzhou, China 


\title{
Experimental instruction in photonics for high school students: approaches to managing problems faced
}

\author{
Zhengyang Choong \\ Hwa Chong Institution (College Section), 661 Bukit Timah Road, 269734
}

\section{Abstract}

Student research projects are increasingly common at the K-12 level. However, students often face difficulties in the course of their school research projects such as setting realistic timelines and expectations, handling problems stemming from a lack of self-confidence, as well as being sufficiently disciplined for sustained communication and experimentation.

In this work, we explore manifestations of these problems in the context of a photonics project, characterising the spectrum of the breakdown flash from Silicon Avalanche Photodiodes. We report on the process of planning and building the setup, data collection, analysis and troubleshooting, as well as the technical and human problems at each step.

Approaches that were found to be helpful in managing the aforementioned problems are discussed, including an attention to detail during during experimental work, as well as communicating in a forthcoming manner. The former allowed for clearer planning and the setting of quantifiable proximal goals; the latter helped in motivating discipline, and also helped in the understanding of research as an iterative learning process without a clear definition of success or failure.

Keywords: experimental instruction, high school research project, student motivation

\section{A photonics high-school project}

\subsection{Background}

Student research projects are increasingly common at the K-12 level; in Singapore, research programmes for high school students are not uncommon (Hwa Chong Institution 2017; Lai et al. 2002), with an ecosystem of competitions (Science Centre Singapore 2017) to boot. This can be explained by a recognition for a shift towards a knowledge-based economy (Yue 2001); the idea of a knowledge-based economy was first mentioned by Peter Drucker in his book The Effective Executive (Drucker

\footnotetext{
14th Conference on Education and Training in Optics and Photonics: ETOP 2017, edited by Xu Liu,

Xi-Cheng Zhang, Proc. of SPIE Vol. 10452, 104524B · C 2017 ICO, IEEE, OSA, SPIE

CCC code: $0277-786 X / 17 / \$ 18 \cdot$ doi: $10.1117 / 12.2266605$
} 
2006). Since 1997, Singapore's education system has oriented itself towards such a knowledge-based economy (Boon and Gopinathan 1965, p. 29), funding talent development programmes such as the Gifted Education Programme and the Music Elective Programme. For instance, the Science Research Programme (SRP) is organised by the National University of Singapore (NUS) in collaboration with local high schools, allowing students at the K-11 level to undertake research projects alongside mentors from universities and other research organisations (National University of Singapore 2017). Each research cycle lasts about a year. As compared to intra-school science research activities, these interactions with external mentors are closer in nature to that of research in academia, with mentors a part of academia themselves.

High school research projects in photonics take place in this environment; Hwa Chong Institution, a high school in Singapore set up a photonics lab more than ten years ago, with attempts to teach photonics concepts (namely polarisation) (Erkan Polatdemir 2015), as well as facilitating research projects in photonics.

\subsection{Project goals and objectives}

The goal of my photonics research project was to build a grating monochromator. A monochromator selects a single wavelength of light from a polychromatic source, typically using a diffraction grating (Loewen, Neviere, and Maystre 1977). This monochromator is aligned to the HeNe laser line at $632.8 \mathrm{~nm}$, with the intention of extending this technical capability to characterise the breakdown flash of avalanche photodiodes at the single-photon level. The research activities in the project were thus to characterise the resolution of such a grating spectrometer, along with its collection efficiency, beam profile, and other related properties.

\section{Obtaining a spectrum measurement within a day}

Research projects usually begin with planning of the setup as well as methodology. At the beginning of the project, I had conceptual difficulties understanding how a blazed diffraction grating worked. Monochromator setups typically use blazed reflective gratings, as they are designed for a higher efficiency at the first order. Specifically, I was stuck on undersatnding an equation that described the relationship between angle of incidence $\theta_{i}$ and the diffracted angle $\theta_{m}$ using the difference between the two angles, although it was clearly equivalent to the typical diffraction equation of $\left(\sin \theta_{m}=\sin \theta_{i}+\frac{m \lambda}{d}\right)$ Eventually, my mentor requested that I attempt aligning the diffraction grating anyway. This led to the prototype that is documented in Figures 1 and 2 .

\subsection{Setting of proximal sub-goals}

This experience was instructional as it was a clear demonstration that substantial progress could be made through the setting of immediate and achievable goals, as 


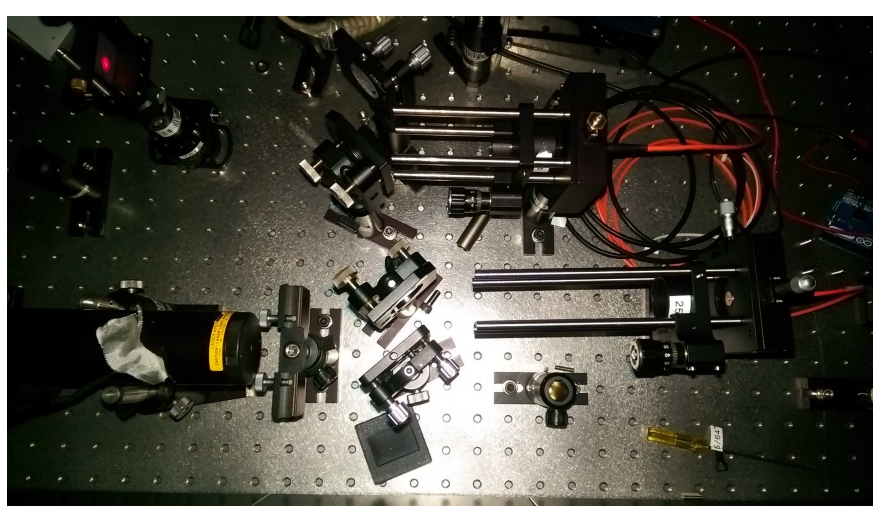

FIGURE 1: Photo of grating setup

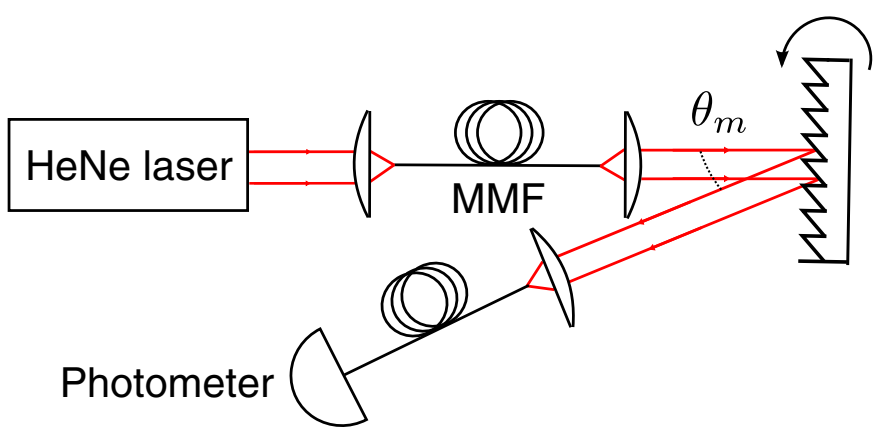

Figure 2: For the calibration setup in Fig. 1, we use a Melles-Griot HeNe laser $(632.8 \mathrm{~nm}, 25-\mathrm{LHP}-925)$ as the light source; coupled into a multi-mode fiber (0.22 NA, Thorlabs M42L02) using a focusing lens (25.0 mm effective focal length, design wavelength $587.6 \mathrm{~nm}$, EdmundOptics 45098). The beam is collimated using similar equipment with the lens mounted on a $x-y$ translation stage. The beam is incident on a blazed reflective grating (500 $\mathrm{nm}$ blaze, 600 lines/mm, Thorlabs GR25-0605), mounted on a motorised rotational stage (Thorlabs CR1-Z7,2.16 arcsecond stepsize). The first diffraction order is coupled into another multi-mode fiber and a photometer. (EdmundOptics Digital Laser Photometer)

compared to goals that lacked a clear deadline and a set of requirements. The setting of immediate, or proximal goals has been shown to have positive motivational effects by providing immediate incentives and guides for improvement (Bandura and Schunk 1981). Goals can affect performance through four mechanisms: directing attention, motivating effort, affecting persistence, and help to lead to the discovery of relevant knowledge and skills. (Locke 2002, p. 3) With these proximal goals, there was thus a sense of urgency and forward momentum, helping to enable and motivate experimental progress.

This preliminary prototyping also provided a reference on which subsequent efforts built on. For instance, having built a prototype for a grating monochromator, I was then able to make further improvements in alignment, swapping one of the 


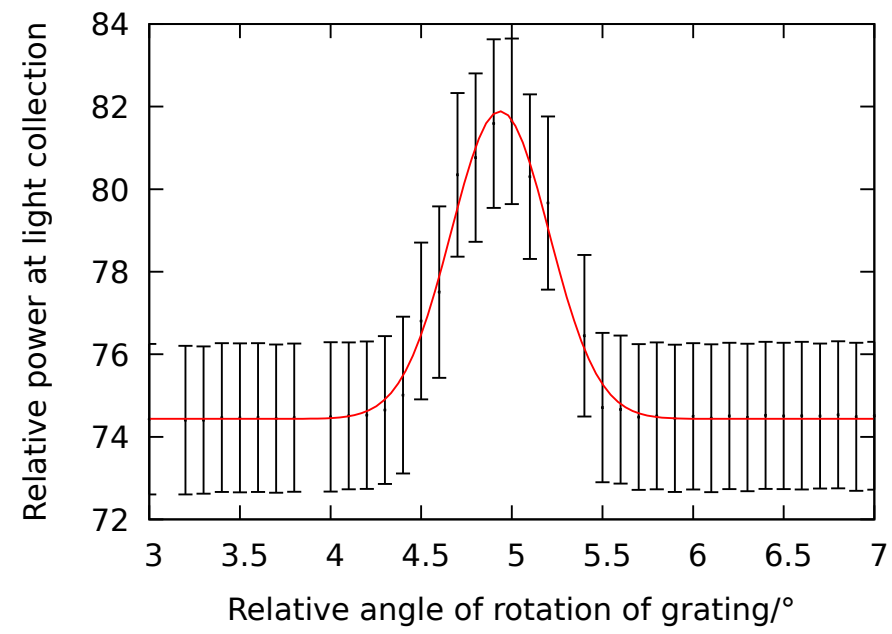

FIGURE 3: Sample plot obtained during preliminary prototyping. Note the relative lack of attention with respect to the error analysis, curve-fitting, as well as the labelling of axes.

multi-mode fibres for a single-mode fibre, as well as to characterise the noise at the light collecting device, and so on.

In the following section, I will examine the implications of this reiteration on an emotional level, bringing in the socio-psychological model as proposed by Carol Dweck et. al.

\subsection{Performance vs. Learning outcomes}

In an intensely competitive education system with multiple high-stakes exams, "exposing students to high-stakes assessments in which there must be necessarily winners and losers ... is likely to produce detrimental effects " (Gregory and Clarke 2003).

This focus on assessments can be understood as a focus on performance goals. Carol Dweck has shown goals to be "central determinants of achievement patterns" (Elliott and Dweck 1988), where two different kind of goals, performance and learning goals, can shape outcomes related to motivation and achievement. Learning goals involves learning at the risk of displaying mistakes, leading to mastery-oriented responses, where "individuals seek to increase their ability or master new tasks". Performance goals, on the other hand, is correlated with a "learned-helplessness response in problem-solving and negative affect”. In a later paper, Dweck explains that:

"Performance goals create a context in which outcomes (such as failures) and input (such as high effort) are interpreted in terms of their implications for ability and its adequacy. In contrast, learning goals create a context in which the same outcomes and input provide information about the effectiveness of one's learning and mastery strategies.”. (Dweck and Leggett 1988) 
These terms help to understand the research experience at the high school level. In an environment that prioritises performance goals that demonstrate student competency, students who have low confidence in their abilities take setbacks badly; this can develop into a sense of helplessness as documented by Dweck; students attribute their failure to a lack of ability, and respond "with negative affect" (Dweck and Leggett 1988; Diener and Dweck 1980). As much as proximal sub-goals may help to frame research problems into achievable and immediate goals, attitudes towards goals as performance or learning based will shape student response. Given that many students do have low confidence in their research abilities - having minimal prior experience - a sense of powerlessness with regards to perceived ability is explainable and understandable.

How then, might we encourage an orientation towards learning outcomes? In Singapore, high school research programmes are formalised to the extent where an entire ecosystem of competition exists, and research is to some extent functionalised. In this light, learning-oriented goals in an environment prioritising assessment and performance seem dichotomous. The interaction between the student researcher and the mentor, a separate environment in itself, is thus particularly important in attempting to cultivate a learning-oriented mindset. In the research process, my mentor would repeatedly emphasise the goal of research as a learning process, as opposed to obtaining results as an end in itself, helping to improve affect.

\section{Emailing as a mode of communication}

Have discussed the manner in which goal-orientation interacts with the research process at a high school level, I will now discuss problems and issues relating to the mentor-mentee interaction with respect to communication. I estimate that there have been at least 500 emails sent over the course of my research project; given that mentors have their own workloads and schedules, it is difficult for them to be physically present, helping to guide experimental work. Thus, emails are used to facilitate the communication of experimental findings, observations, and data. Emailing is categorised under the broader label of "Computer-Mediated Communication" within literature. (Walther 1992; Walther 1996; Dubrovsky, Kiesler, and Sethna 1991); I would like to discuss their content, with regards to what makes for effective communication, and also examine the broader socio-emotional implications that comes with emailing.

\subsection{Attention to detail}

In the course of emailing, one skill that was repeatedly emphasised was an attention to technical detail in planning, which included an awareness for scheduling, as well as setting proximal sub-goals: laying out the individual steps required for a certain goal. This provides a structured framework which can then be referenced to during experimental work. This is particularly pertinent in photonics projects, where

An excerpt of such a plan is provided below: 
second iteration: tear down the first one. build a basic michelson interferometer again, but now:

- add two linear polariser and laser line filter. this implies aligning the linear polarisers to each other first.... and is worth another thread / email on this

- check that the $632.8 \mathrm{~nm}$ hene laser polarised? (i remember that it is)

- the beamsplitter is polarising too? (yes) is it roughly 50:50 for different polarisations? what happens if i enforce a vertical polarisation throughout?

- use washers with $1 / 4$ " screws

- connect the arduino to the photometer and let it log to a file. i might decide this arduino to connect to the spectroscope pc tentatively, since there isn't any spare pc in the lab. assuming the photometer is callibrated.... as long as it passes the sanity check i think it's fine

This was a plan for building a prototype meant to perform Fourier spectroscopic measurements. With a structured framework, there are immediate motivations and indicants of self-mastery. (Bandura and Schunk 1981). Communicating these plans helps to formalise the procedure, as well as to obtain feedback at various junctures of execution.

Not discounting the utility of emails in helping to facilitate mentor-mentee interaction, along with preparing one for experimental work, I have personally found it an emotionally-taxing time sink. Aside from the interaction between low confidence and an orientation towards performance goals as described in the section above, it seemed as if, during the course of the research that emailing started to feel a little impersonal.

since you guys are now barred from the lab, take the time to finally get other basics in working order today's homework (do on your own, stop copying from each other) give me a perfect and proper plot for any dataset, can be randomly generated for all i care at the moment.

since you guys need deadlines and unkind pressure to move you have until $6 \mathrm{pm}$ to do so.

To provide some context, there were two other students in the research group; they had difficulties communicating with the mentor over the course of the research cycle. The mentor-mentee communication started in May, with the research work meant to end the following January. The above excerpt was sent in the middle of November, at 4:41 pm, and was at that point mildly stress-inducing. In retrospect, the instructions and requests were fairly reasonable, and were valuable pieces of instruction in data plotting and the interpretation of fit statistics. Put into context however, 
it sounded harsh and felt excessively demanding. The following section will discuss how electronic communication reduces the number of social cues, which can make communication appear more impersonal.

\subsection{Computer-mediated communication as impersonal}

It has been noted that emailing, under the category of 'Computer-mediated communication', can be seen as impersonal. (Walther 1996). It is suggested that the lack of nonverbal social cues present in electronic communication as compared to face-toface meetings leads to more impersonal interaction. (Hiltz, Johnson, and Turoff 1986) Emailing separates the human from the task, and although this does appear to improve task quality (see Hiltz et. al), there are fewer avenues for the diffusion of tension, the communication nuanced expectations and statements, leading to emails that are perhaps a little blunt.

It is important to realise that communication is not an end in itself; it is meant to facilitate mentor-mentee interaction, given that mentors are often busy. Writing emails with detailed plans, schedules, and contingencies, better prepares one for experimental work. Not discounting its importance, in the context of the high school research programme, emailing should perhaps a degree of restraint, with more mentor-mentee interaction taking place via face-to-face interaction. In the following section, I will discuss the research experience as it was closer to the end of the research cycle, involving data collection and report writing.

\section{Data collection and error analysis}

Having discussed the process of planning and emailing, I will now discuss experiences with respect to experimental work and data analysis, as well as the weekly meetings that took place near the end of the research cycle. Although a detailed experimental plan that takes into account proximal sub-goals provides some confidence as to the feasibility of the research work, executing the plan is a different issue. There are some ideas important in data collection that do not seem to be included in the formal education system, which in turn affects experimental work.

K-12 Science education in Singapore involves some degree of hands-on learning and experimentation (Ministry of Education, Singapore 2013). However, the standards of experimentation of that in school and actual research differ significantly with respect to the treatment of experimental certainty, as well as data analysis. School practicals tend to limit themselves to three or so observations at each data point for practical reasons, given a lack of automation via computer scripting. Lacking formal training in the use of statistics for scientific means, there are limited experiences with regards to the experimental fitting of data to a theoretical equation, taking into account various

fit statistics such as $\chi^{2}$ and the degrees of freedom in a fit. In the email excerpt quoted above on plotting, the discussion turned out to be instructional with respect to what plot statistics meant. 


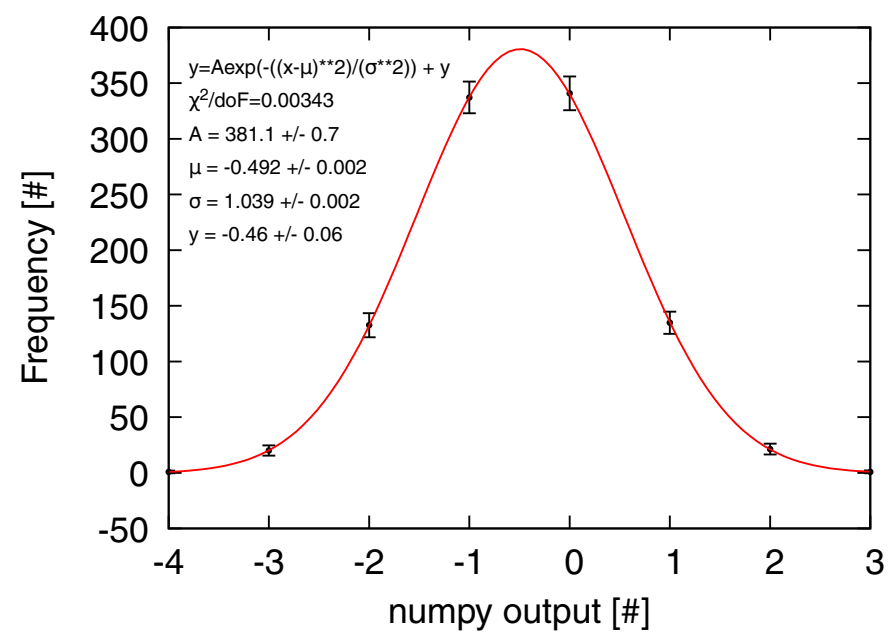

Figure 4: The Python package numpy was used to generate a set of pseudorandom numbers that follow a Gaussian distribution; the data was binned and fitted to such a Gaussian distribution. The error estimators are the standard deviation at each point. Having the fit equation and statistics inside the plot makes them easier to interpret. The $\chi^{2}$ statistic suggest overfitting, where the error estimators used here are too large.

At the same time, it is important to manage expectations of experimental work, especially in a performance-oriented environment, and take things as they come. For instance, during the process of data collection, there was the impression that there existed a "correct data": in measuring the spectrum of the HeNe laser using the grating spectrometer, I have obtained data suggesting that the specific HeNe tube used had an additional spectral peak at $633.9 \mathrm{~nm}$ alongside the main $632.8 \mathrm{~nm}$ emission peak, for which I have not yet found an explanation for. I have tried performing similar measurements on three other laser sources, and it appears that this additional artefact is specific to the laser tube I was using.

At the same time, when performing a spectral scan, the grating was rotated with a smaller stepsize so as to obtain a higher resolution scan; it has revealed several interesting features, for which there is no obvious explanation. I have tried averaging over several scans to see if it were a result of noise, as well as correlating separate scans without reaching a satisfactory conclusion. When this happened, it felt as if this investigation was only tangentially related to the "main goal", which deviated from the notion of "correct data". I would suggest that it is precisely these interesting features that are worth investigation and further study. If the data we obtain merely serve to confirm our own biases, then perhaps there has to be a change in our approach and methodology.

The idea of "correct data" involves the assumption that everything will work as expected, which is not particularly useful in a research setting. The management of expectations in the face of failure is thus particularly important, as research is an iterative task; the distinction between performance and learning goals is thus relevant: an orientation towards performance-goals influences one to work towards their own 


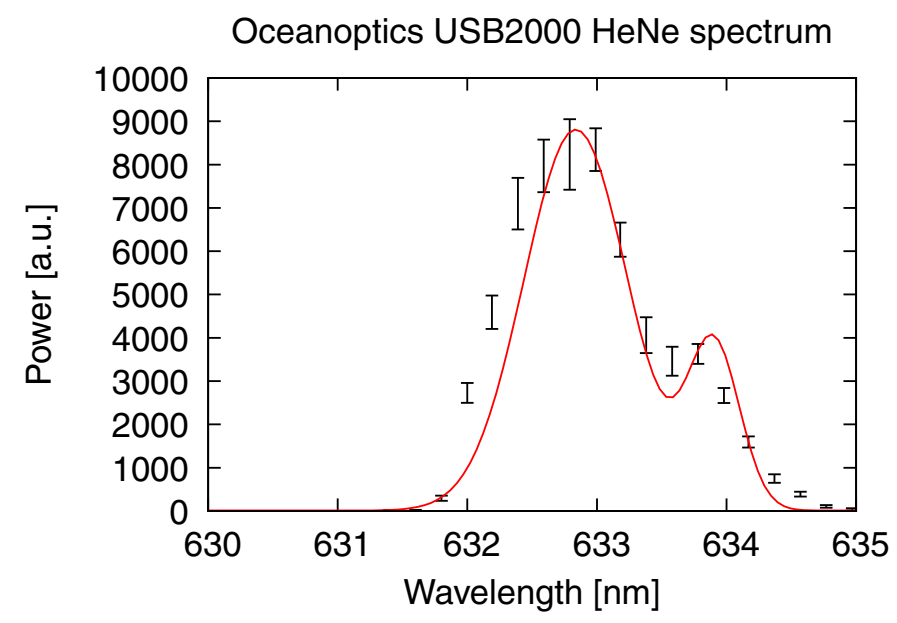

Figure 5: HeNe laser spectrum as measured by the Oceanoptics USB4000 spectrometer, fitted to a bi-Gaussian. Error-bars are the computed standard deviation at each point.

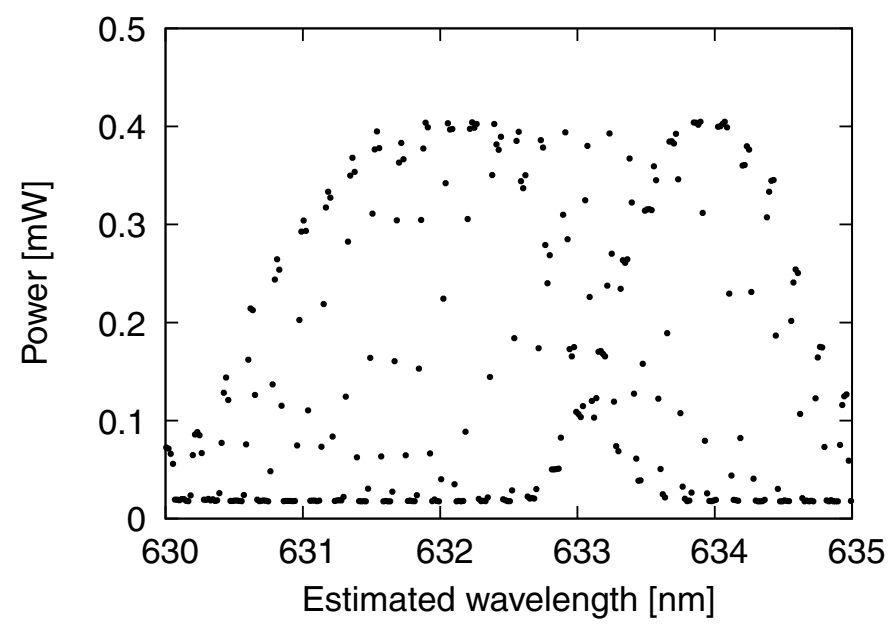

FIGURE 6: HeNe spectra as measured by grating setup. These peaks have a FWHM of less than $0.1 \mathrm{~nm}$, suggesting that these features are artifacts, as the grating cannot resolve details beyond $0.1 \mathrm{~nm}$, the theoretical resolution limit.

biases of what data "should" be observed.

In conclusion, experimental work comes with many uncertainties, both human and technical, and it is important to learn how to live with them. During this period of experimentation, I found that I was under time pressure to wrap up the research project, which led to a focus on the data I "expected" to see, rather than exploring the real unknowns that I faced in the course of experimental work. This could have perhaps been better dealt with, should the process of data collection have started earlier; the manner in which schedules and goals are set is thus relevant in time management. 


\section{Conclusion}

We have thus examined issues pertaining to goal-setting and motivation, communication, and data analysis. The research experience is not meant to be smooth-sailing, and I think that a training in research skills develops skills tangentially related to the academic curriculum. The findings can thus be summarised to the following points: (1) the setting of proximal goals that are specific, immediate and achievable guides experimental work; (2) the nature of the goals set as performance or learning oriented will influence both learning outcomes and affect; (3) electronic communication is inherently impersonal as there are relatively fewer social cues, possibly leading to lower affect.

The nature of high school projects in photonics is such that the high school syllabus is inadequate in its instruction of statistical and experimental techniques. At the same time, the orientation of the school mindset towards that of performance goals is not necessarily a healthy one. Therefore, communication and goal-setting are of particular importance in experimental instruction: helping to manage expectations, dveloping a sense of intuition, as well as managing failure in a competitive and performanceoriented environment.

\section{Acknowledgements}

I would like to express my gratitude to my parents who have always supported my journey, wherever that leads. I would also like to thank Dr. Erkan Polatdemir and Mrs. Tan Ai Chin from Hwa Chong Institution for providing assistance for my attending the ETOP2017 conference. Lastly, I am grateful to my mentor, Dr. Tan Peng Kian from the Centre of Quantum Technologies (National University of Singapore), for having provided guidance through these years.

\section{References}

[1] Albert Bandura and Dale H Schunk. "Cultivating competence, self-efficacy, and intrinsic interest through proximal self-motivation." In: Journal of personality and social psychology 41.3 (1981), p. 586.

[2] GC Boon and S Gopinathan. "The development of education in Singapore since 1965". In: Toward a better future: Education and training for economic development in Singapore since (1965), pp. 12-38.

[3] Carol I Diener and Carol S Dweck. "An analysis of learned helplessness: II. The processing of success." In: Journal of personality and social psychology 39.5 (1980), p. 940.

[4] Peter F. Drucker. The Effective Executive. Revised. Harperbusiness Essentials. Harper Paperbacks, 2006. 
[5] Vitaly J Dubrovsky, Sara Kiesler, and Beheruz N Sethna. "The equalization phenomenon: Status effects in computer-mediated and face-to-face decision-making groups". In: Human-computer interaction 6.2 (1991), pp. 119-146.

[6] Carol S Dweck and Ellen L Leggett. "A social-cognitive approach to motivation and personality.” In: Psychological review 95.2 (1988), p. 256.

[7] Elaine S Elliott and Carol S Dweck. "Goals: An approach to motivation and achievement.” In: Journal of personality and social psychology 54.1 (1988), p. 5.

[8] P. K. Tan Erkan Polatdemir. Learning Polarisation of Light in a Student-centred Learning Environment. 2015. URL: https : / / www . rp . edu .sg/uploadedFi les/Contents /RP/Schools_And_Centers /Centre_For_Educational_ Development/05Research/02Newsletter/FA_ReflectionsOnPBL_De c2015_NoBleed.pdf.

[9] Kelvin Gregory and Marguerite Clarke. "High-stakes assessment in England and Singapore”. In: Theory into practice 42.1 (2003), pp. 66-74.

[10] Starr Roxanne Hiltz, Kenneth Johnson, and Murray Turoff. "Experiments in group decision making communication process and outcome in face-to-face versus computerized conferences". In: Human communication research 13.2 (1986), pp. 225-252.

[11] Hwa Chong Institution. Hwa Chong Centre for Talent Development. 2017. uRL: h ttp://www.hci.edu.sg/advantage/talent-development/centad.

[12] T. Lai et al. "Establishing a photonics teaching facility and program in a Singapore secondary school”. In: Seventh International Conference on Education and Training in Optics and Photonics. Ed. by T. K. Lim and A. H. Guenther. Vol. 4588. May 2002, pp. 120-130. DOI: 10.1117/12.468689.

[13] Gary P. Locke Edwin A.; Latham. "Building a practically useful theory of goal setting and task motivation: A 35-year odyssey." In: American Psychologist 57 (9 2002). DOI: $10.1037 / 0003-066 X .57 .9 .705$.

[14] EG Loewen, M Neviere, and D Maystre. "Grating efficiency theory as it applies to blazed and holographic gratings”. In: Applied optics 16.10 (1977), pp. 27112721.

[15] Ministry of Education, Singapore. Science Syllabus - Lower Secondary. 2013. uRL: https : / / www . moe.gov.sg/docs/default-source/document/educ ation/syllabuses/sciences/files/science-lower-secondary$2013 \cdot p d f$.

[16] National University of Singapore. Science Research Programme (SRP). 2017. URL: http://www. science. nus . edu.sg/outreach/srp-menu.

[17] Science Centre Singapore. The Singapore Science and Engineering Fair. 2017. URL: http://www.ssef.com.sg/ssef/. 
[18] Joseph B Walther. "Computer-mediated communication: Impersonal, interpersonal, and hyperpersonal interaction”. In: Communication research 23.1 (1996), pp. 3-43.

[19] Joseph B Walther. "Interpersonal effects in computer-mediated interaction a relational perspective”. In: Communication research 19.1 (1992), pp. 52-90.

[20] Chia Siow Yue. "Singapore: towards a knowledge-based economy". In: Industrial restructuring in East Asia: Towards the 21st century 25 (2001), p. 169. 